\title{
TRATAMIENTO DEL PROLAPSO DE CUPULA VAGINAL POR LA COLPOCISTOPEXIA VIA ABDOMINAL
}

\author{
Doctores Alvaro Fonnegra Miramón * y Rubén Darío Guzmán Alandete **
}

El prolapso de cúpula vaginal después de histerectomía vaginal o abdominal, es un problema de difícil solución.

Se han propuesto diversas técnicas para corregir esta procidencia: la de Brady quien preconiza la fijación de la cúpula a la pared abdominal anterior; la de Grand-Ward quien describió una operación utilizando fascia de buey; la de Norman Guiou utilizando fascia lata insertada en la bóveda vaginal; la promonto-cistopexia o sea el método que fija la cúpula al promontorio $u$ operación de Normal Miller.

En nuestro medio el profesor Héctor Enrique Bernal preconiza una técnica por vía vaginal, formando arbotantes de la bóveda vaginal y en que se da primordial importancia a la cura del enterocele.
En 1948 Paul Fletcher describió una técnica llamada colpocistopexia utilizando tiras de fascia abdominal cruzándolas y usándolas como tejido suspensor de la cúpula. Al propio tiempo Williams y Richardson describieron una muy similar que consistía en la utilización de tiras aponeuróticas de los oblicuos externos que se conducían por vía extraperitoneal siguiendo el curso de los ligamentos redondos e insertándolas a la bóveda vaginal.

Entre nosotros no existe ninguna publicación hasta la fecha sobre la corrección del prolapso de cúpula vaginal por vía abdominal utilizando colgajos de tejido aponeurótico de los oblicuos externos y fascias subyacentes. Solamente algunos cirujanos han practicado esta operación en casos aislados, sin reunir una casuística suficiente como

\footnotetext{
* Profesor Asociado de Ginecología.

** Instructor de Ginecología.

Del Departamento de Obstetricia y Ginecología.

Hospital San Juan de Dios - Facultad de Medicina - Universidad Nacional de Colombia. Bogotá, D. E., 1963
} 
para valorar la bondad de dicho tratamiento.

Al respecto presentamos una serie de 22 casos de colpocistopexia para el tratamiento del prolapso de la cúpula vaginal, utilizando tiras aponeuróticas de los oblícuos externos, según técnica que detallamos más adelante con algunas modalidades y variantes de acuerdo con los casos.

\section{Material y métodos}

La mayor parte del material humano procede del Hospital San Juan de Dios, de Bogotá (14 casos) operados a partir de 1952, hasta la fecha; 3 casos de pacientes del ICSS y 5 de la clientela particular.

Por ser esta intervención de difícil ejecución, todas las intervenciones quirúrgicas fueron realizadas por personal calificado (profesores e instructores) en lo que al Hospital se refiere y por ese mismo personal en la clientela particular y del ICSS.

El material se escogió de enfermas a quienes se les había practicado histerectomía abdominal total o vaginal por causa que justificase tal conducta, como se verá en los cuadros adjuntos. Hay que anotar que de los 14 casos de San Juan de Dios, solamente dos fueron intervenidos inicialmente en esa institución, pues los 12 restantes procedían de otros sitios y consultaron por su sintomatología de prolapso.

Seguimos el siguiente procedimiento quirúrgico:
Antes de practicar la laparotomía se lava cuidadosamente la vagina con agua y jabón y se introduce una bujía de las que se usan para dilatación uterina de más grueso calibre fijándola con esparadrapo con el objeto de que no se caiga; esto para que al abrir la cavidad se pueda identificar fácilmente la cúpula. Luego se comienza la laparotomía.

Una vez abierta la piel y el tejido celular subcutáneo, por incisión de Phanestiel preferentemente, se limpia cuidadosamente la aponeurosis del oblicuo mayor del abdomen en un espacio suficiente; luego se diseca una tira a cada lado de la aponeurosis del oblícuo mayor de 2 centímetros de ancho aproximadamente por 8 a 10 centímetros de longitud. Dichas tiras se pueden cortar en sentido longitudinal o transversal, pero se prefieren oblícuas, con base inferior cuando el colgajo es longitudinal y con base superior cuando se oblicua o transversa y aproximadamente a 1 centímetro por encima del correspondiente orificio del anillo inguinal externo. Desde luego las porciones de las tiras deben contener fibras reforzantes del oblicuo menor; luego de completar la incisión de Phanestiel y separar los músculos rectos a los lados, se abre el peritoneo y se identifica el vértice de la vagina que se ha separado con la bujía. Acto seguido se introducen las tiras aponeuróticas ya separadas, siguiendo el curso de los ligamentos redondos o sea a través del anillo inguinal. Al llegar al orificio interno se pasan extraperitoneales con la ayuda de una pinza larga roma, hasta alcanzar la parte lateral de la cúpula vaginal. Si se encuentra alguna dificultad técnica para conducir 
los colgajos extraperitonealmente, se colocan dentro de la cavidad abdominal cerca al peritoneo y se practica en éste una abertura en forma de pestaña con el objeto de cubrir el colgajo aponeurótico o aplicando directamente el peritoneo sobre la tira.

Luego de abrir la hojilla peritoneal que recubre la cúpula se rechaza ésta de la vejiga para dar sitio donde han de implantarse las tiras. Aconsejamos como modalidad muy importante y siempre y cuando se trate de una vagina de suficiente capacidad, recortar un manguito de cúpula de aproximadamente 2 cms de longitud y cerrarla con puntos no perforantes e invaginantes, antes de la implantación de los colgajos, los cuales se entrecruzan y se fijan a la parte anterior de la vagina reforzándolos entre sí y usando material de seda fina con puntos no perforantes, o catgut cromado número 1 . Cuando existe cistocele, que es lo frecuente, se aplican cuatro puntos laterales a cada lado de la fascia de vejiga adosándola a las tiras y a la vagina ya recortada y suturada. Luego se cierra el peritoneo de la cúpula vaginal, se retira el mandril de la vagina y se procede a practicar el procedimiento de Moscowitz (cierre del douglas con una serie de suturas en bolsa) con el objeto de corregir el enterocele. Una vez cerrada la cavidad abdominal por los métodos clásicos, se procede a practicar una perineorrafia complementaria para darle mejor sostén al piso pélvico y corregir el rectocele.

Siguiendo las normas aconsejadas en este trabajo hemos conseguido una cura del prolapso de la cúpula vaginal posthisterectomías en todas las enfermas controladas por nosotros, desde el segundo mes postoperatorio la de menor control, hasta diez años la de máximo control.

\section{CONCLUSIONES}

1. Se presentan 22 casos de enfermas con prolapsos de cúpula vaginal tratados por medio de la colpocistopexia vía abdominal utilizando sendos colgajos de aponeurosis de los oblícuos externos como tejido suspensor de la cúpula y complementándola en la mayoría de los casos con el procedimiento de Moscowitz y perineorrafia.

2. Casi todo el material humano procede del Hospital San Juan de Dios de Bogotá (14 casos), además de 3 casos de enfermas del ICSS y 5 casos de la clientela privada.

3. Esta intervención debe ser practicada por personal especializado, por requerir su ejecución una técnica rigurosa.

4. Se encontró muy poca morbilidad y la mortalidad fue nula.

5. De los 22 casos tratados con esta técnica se controlaron 15 , sin encontrarse en ninguno de ellos reproducción del prolapso.

6. Aconsejamos este procedimiento con algunas de sus modalidades técnicas como tratamiento de elección en los casos de prolapso de cúpula vaginal concomitantes o no con procidencia de vejiga, recto o enterocele. 
B I B L I $O O$ G

1. Tratado de técnica quirúrgica. Profesor Greenhill.

2. Enciclopedia médico-quirúrgica. Ginecología, 1961.

3. Indices de nomenclatura standard; Asoc. Med. Amer. y clasificación estadística. O.I.N.S. Hospital San Juan de Dios de Bogotá.

4. BERnAL B., HECTOR E.: El prolapso de la cúpula vaginal. Rev. Col. de Obst. y Ginecología. 394-427, julio y agosto de 1961.

5. BRADY LEO: Operation to correct genital prolapse following vaginal panhysterectomy. Am. J. Obst. \& Gynec. 32: 295-299, 1936.

6. FLETCHER PAUL F.: Abdominal colpocytopexy for complete prolapse of the vagina and bladder. Am. J. Obst. \& Gynec. 56: 41, 1948.

7. MILLER NORMAN F.: New method of correcting complete inversion of vagina with or without complete prolapse, two cases.Surg. Gynec. \& Obst. 15: 7-21, 1912.

8. GUIOU NORMAN M.: Fascial suspentiom of the vagina. Canadian M. A. J. 60: $600,1949$.

9. MoschCowitz A. V.: Surg. Gynec. \& Obst. 15: 7-21, 1912.

10. RODRIGUEZ SOTO ARTURO. Histerectomías. Mil operaciones consecutivas. Rev. Col. de Obst. y Ginecología, 295-322, julio y agosto de 1961.

11. TE-LINDE RICHARD: Ginecología Operatoria, 1961.

12. WARD G. E.: Arch. Surg. 36: 163-170, 1938.

13. WILLIAMS GEO A., RICHARDSON A. CULLEN: Transplantation of external oblique aponeurosis; an operation for prolapse of the vagina following hysterectomy. 


\section{TRATAMIENTO DEL PROLAPSO DE CUPULA VAGINAL POR LA COLPOCISTOPEXIA VIA ABDOMINAL}

\section{PROLAPSO DE LA CUPULA VAGINAL 22 CASOS}

Edad: 38-63 años

Antecedentes quirúrgicos
Histerectomía vaginal:
Histerectomía abdominal:
13 casos
Histerectomía radical:
8 casos
1 caso

Paridad: $2-15$

Síntoma inicial: Sensación de cuerpo extraño en genitales externos

\section{HALLAZGOS CONCOMITANTES}

Uretrocistocele

Rectocele y/o desgarro perineal

Enterocele

$$
\begin{aligned}
& \text { I : } 6 \\
& \text { II : } 4 \\
& \text { III : } 4
\end{aligned}
$$

$$
\begin{array}{r}
\text { I } \\
\text { II }
\end{array}
$$$$
\text { III : } 1
$$

Incontinencia urinaria

$6 \quad$ Fístula vesico-vaginal 1

Procedencia ano-rectal

1

COLPOCISTOPEXIA POR VIA ABDOMINAL

Modalidades de técnica

Acortamiento por sección de cúpula 14 Abertura o plicatura peritoneal 4 Moscovich

Perineorrafia

Accidentes operatorios

Anestesia

Abertura de vejiga

General

Raquídea

Transfusión sanguínea

Complicaciones postoperatorias

$\begin{array}{lr} & \text { Caso } \\ \text { Abertura de vejiga } & 2 \\ \text { General } & 16 \\ \text { Raquídea } & 6 \\ & 3\end{array}$

Hematoma y/o absceso de cúpula

Hematoma y/o absceso de pared

COLPOCISTOPEXIA POR VIA ABDOMINAL

Estadía hospitalaria

Morbilidad febril

Mortalidad

Controles
10 a 34 días

8 casos

0

$\begin{array}{lrl} & 22 \text { días } \\ \text { Máximum } & 8 \text { días }\end{array}$

\begin{tabular}{rrrlrl}
2 & a & 6 & meses & 7 & casos \\
6 & a & 12 & meses & 4 & casos \\
2 & a & 5 & años & 2 & casos \\
5 & a & 10 & años & 2 & casos \\
& \multicolumn{1}{r}{ Total: } & 15 & casos
\end{tabular}

No se ha presentado reproducción del prolapso en ninguno de los casos controlados 